\title{
BMJ Open Supervision training interventions in the health and human services: realist synthesis protocol
}

\author{
Sarah Lee, ${ }^{1}$ Charlotte Denniston, ${ }^{1,2}$ Vicki Edouard, ${ }^{1}$ Claire Palermo, ${ }^{1}$ Kirsty Pope, ${ }^{3}$ \\ Keith Sutton, ${ }^{4}$ Susan Waller, ${ }^{4}$ Bernadette Ward, ${ }^{5}$ Charlotte Rees ${ }^{1}$
}

To cite: Lee S, Denniston C, Edouard V, et al. Supervision training interventions in the health and human services: realist synthesis protocol. BMJ Open 2019;9:e025777. doi:10.1136/ bmjopen-2018-025777

- Prepublication history for this paper is available online. To view these files please visit the journal online (http://dx.doi org/10.1136/bmjopen-2018025777).

Received 1 August 2018 Revised 14 March 2019 Accepted 12 April 2019

Check for updates

(C) Author(s) (or their employer(s)) 2019. Re-use permitted under CC BY-NC. No commercial re-use. See rights and permissions. Published by BMJ.

${ }^{1}$ Faculty of Medicine, Nursing and Health Sciences, Monash Centre for Scholarship in Health Education (MCSHE), Monash

University, Melbourne, Victoria, Australia

${ }^{2}$ Department of Medical

Education, Melbourne Medical

School, The University of

Melbourne, Melbourne, Victoria,

Australia

${ }^{3}$ Department of Occupational

Therapy, Monash University,

Frankston, Victoria, Australia

${ }^{4}$ Rural Health, Monash

University, Warragul, Victoria, Australia

${ }^{5}$ Rural Health, Monash University, Bendigo, Victoria, Australia

Correspondence to

Miss Sarah Lee;

sarah.lee1@monash.edu

\section{ABSTRACT}

Introduction Supervision training aims to develop workplace supervisory competencies. Despite extensive supervision literature, including literature reviews, the processes through which supervision training interventions produce their effects, for whom and under what circumstances is not clearly delineated. The purpose of this study is to explain the effect of contextual factors on the underpinning mechanisms of supervision training outcomes.

Methods and analysis We propose to examine supervision training interventions across the health and human services workforce using realist methods. Pawson's five stages for undertaking a realist synthesis will be followed: (1) clarifying the scope of the review; (2) determining the search strategy; (3) study selection; (4) extracting data and (5) synthesising the evidence and drawing conclusions. Extracted data will include study characteristics, characteristics of participant cohort, intervention type, contextual factors, underlying mechanisms and supervision training outcomes. Patterns in context-mechanism-outcome configurations will be identified. Initial programme theories will be developed based on a comprehensive search of the literature, which will include key terms relating to supervision and training. The search strategy will involve: (1) electronic database searching using Medline, Cumulative Index to Nursing and Allied Health Literature, Social Services Abstracts, Educational Resources Information Center, PsycINFO and Australian Public Affairs Information Service and (2) hand and citation searching. We will also contact authors where necessary and discuss identified literature among the project team with extensive expertise in supervision training.

Ethics and dissemination The realist synthesis will propose an evidence-informed theory of supervision training interventions (ie, what interventions work for whom and why). The findings will be disseminated in peer-reviewed journals and presentations and through discussions with relevant organisations and stakeholders. The research will be used by educators to develop evidenced-based supervision training interventions. It will also help workplace supervisors to better understand what types of supervision training might work most optimally for them and their colleagues. Other researchers could use the synthesis findings to guide future supervision research.

PROSPERO registration number CRD42018094186

\section{Strengths and limitations of this study}

- This synthesis will identify how and why components of supervision training are effective (or not) within various settings in the health and human services workforce rather than merely focusing on whether supervision training is effective.

- A large multidisciplinary research team lends high-quality relevance and rigour checks and more comprehensive data interpretation.

- The realist synthesis is well suited to the evaluation of complex interventions such as supervision training.

- Only English language studies will be included, so findings might lack transferability to supervision training interventions in non-English speaking countries.

- Search terms for supervision that are less frequently used in the health and human services (eg, critical companion, coaching, facilitation, practice development) may be omitted, meaning that some evidence may be missed.

\section{INTRODUCTION}

Supervision is a distinct professional activity characterised by the training and professional development of supervisees, ${ }^{12}$ and in some literature is understood to be partly hierarchical and evaluative. ${ }^{3}$ Although the definition of supervision varies in terms of its purposes, arguably one of the most widely adopted models describing the purpose and functions of supervision is that of Proctor, ${ }^{4}$ who outlined three functions of supervision: normative (managerial), formative (educational) and restorative (supportive). The normative function depicts the role that supervision plays as a process for ensuring ethical, acceptable and quality practice. ${ }^{5}$ The formative function of supervision encompasses developmental aspects such as gaining knowledge, communication skills, trust in self and confidence. ${ }^{6}$ The restorative function focuses on providing support to alleviate burnout and stress. ${ }^{7}$ All three functions 
highlight supervision as a protective factor in professional practice. ${ }^{8}$ Note that Proctor's depiction of supervision has been used in this study to help guide our understanding and definition of supervision.

A guideline for what constitutes effective supervision for both the supervisor and supervisee has been outlined in the literature. ${ }^{9}$ Effective supervision has been described to include but is not limited to: supervisees choosing their own supervisors ${ }^{10}$; deciding on the most suitable types of supervision (eg, one-to-one or peer group supervision or a combination of both $)^{11}$; establishing a supervision agreement or contract and using a supervision agenda ${ }^{12}$; choosing venues away from supervisees' workplaces to conduct supervision sessions; having an optimal meeting length and frequency (eg, at least 1 hour every 4 weeks) $)^{13}$; using effective communication and feedback ${ }^{14}$; facilitating reflective practice; use of more than one mode for distance supervision; building a positive supervisory relationship (eg, one that is positive, supportive, trustworthy, non-judgmental and encouraging) ${ }^{1012}$; separating clinical supervision from line management; undertaking training in supervision and evaluating supervision (either through formal or informal methods) ${ }^{915}$

Effective supervision is that which has positive outcomes across all levels of an organisation, ${ }^{16}$ including for the client/patient (eg, improved client/patient outcomes) and the organisation as a whole (eg, improved standards and quality of service delivery). ${ }^{9}{ }^{12}$ Benefits of effective supervision for practitioners include developing capability and knowledge ${ }^{17}$ reducing burnout ${ }^{18}$ and assisting practitioners to cope better with their work and workplace. ${ }^{13}$ When conducted effectively, supervision can provide an opportunity for practitioners to reflect on their practice and establish new approaches to supervision. ${ }^{9}$ While effective supervision and its outcomes have been clearly delineated in the literature, ${ }^{19-21}$ the same attention has not been afforded to what constitutes effective training for supervisors. ${ }^{22}$

This realist synthesis protocol focuses on supervision practised in both the broad domains of health and human services given that it is part of a broader programme of research investigating supervision training in these wideranging settings including: health, housing, children services, youth and family services, alcohol and drug services and mental health. Although Proctor's model of supervision has been used to guide our understanding of supervision in the health and human services, any outcomes of supervision training will be explored in this study including both positive and negative outcomes at individual, interpersonal and organisation levels. What follows is a brief outline of: (1) the characteristics of supervision within these settings and (2) the different modes of supervision training commonly implemented to support supervisors within these settings.

\section{Supervision in the health and human services}

Literature within health and human services predominately describes supervision as an educative process. For the most part, the literature embraces the formative and restorative functions of Proctor's model, thereby characterising supervision as both a platform for practitioners to develop knowledge and a method of professional support. ${ }^{18}{ }^{23}$ However, within mental health settings, the normative function of supervision is typically privileged, with supervision considered as an important factor in ensuring staff ability and enabling best practice outcomes for consumers and carers. ${ }^{24}$ Other allied health professions, such as physiotherapy and speech therapy, report using discipline-specific supervisory models for a combination of normative, formative and restorative functions. ${ }^{102526}$

In terms of the human services workforce, in some areas of the workforce like alcohol and other drug services, the empirical evidence for the function of supervision is sparse. ${ }^{27}$ Supervision for human services workers appears more complex due to the vast array of diverse settings in which these professionals work. Consequently, there seems to be a multiplicity of definitions, models and applications of supervision within this literature, ${ }^{28} 29$ mirroring the broader supervision literature discussed above.

\section{Supervision training}

Supervision training is a common way to support supervisors and prepare them for their supervisory roles within health and human services. Supervision training is typically a process for enhancing supervisor behaviour to improve the outcomes of the supervisory process. $^{30}{ }^{31}$ Current literature suggests that there is a variety of training methods used to support the development of effective supervisors. ${ }^{32}{ }^{33}$ Supervision training can be in the form of formal training (eg, face-to-face or online workshops and/or seminars), training received informally (eg, role modelling and observation) and/ or self-directed learning (eg, guided reading) ${ }^{33}$ Supervisors typically receive little or no formal training for their supervision role and little is known about how supervisors acquire knowledge and aptitude in the supervisory role. ${ }^{33}$ Supervisor training is rarely empirically or theoretically grounded, ${ }^{3}$ and it appears that most professionals learn supervisor skills by assimilating their own experiences as supervisees or through informal methods (eg, observation). The process of learning to be a supervisor therefore often occurs by default. ${ }^{34}$

Inadequate attention to supervision outcomes, supervision training and supervision research, however, has started to raise concerns about the quality of supervision delivered across a number of different disciplines. ${ }^{27}$ 35-37 The assumption that experience as a practitioner or a supervisee translates into becoming an effective supervisor is, of course, erroneous. ${ }^{38}$ Indeed, there is a distinction between practitioner skills and supervisor skills, ${ }^{33}$ and specific training to develop supervisory skills is crucial.

While there is broad understanding about what constitutes effective supervision, ${ }^{19-21}$ little attention has been paid to what constitutes effective training of supervisors. $^{22}$ Therefore, insufficient clarity still exists 
regarding how supervisors should be trained to become effective supervisors. ${ }^{33}$ It has been said that 'research on the effectiveness of supervision training remains virtually non-existent' (p219). ${ }^{39}$

Three reviews (two systematic, one narrative) regarding supervision training have been published since $2004,,^{33} 30$ and these provide a useful starting point to understand supervision training. First, Gonsalvez and Milne ${ }^{38}$ conducted a narrative review of clinical supervision training changes in professional psychology within Australia, both examining and addressing solutions to emerging problems. Gonsalvez and Milne identified a need for better regulation of supervisory practice within psychology and recommended that supervisory practice be supported with enhanced development of resources (eg, manuals, workbooks and better psychometric tools to evaluate supervisory processes). Second, Milne $e t a l^{33}$ conducted a systematic review of controlled trials of supervision interventions and found clear empirical support for supervisor training; training which when effective could help to bridge the gap between policy and practice and ensure that therapies within the mental health field are implemented with fidelity. This review also helps us to understand the complexity and diversity of supervisor training. For example, they found 56 supervisor-training methods were used across the 11 studies reviewed. Training methods included workshops, role play, modelling, guided reading, direct observation and/or teaching (through verbal instruction). Finally, Tsutsumi's ${ }^{40}$ systematic review, including seven controlled studies assessing the effect of supervisor training on the mental health of subordinate workers, proposed an evidenced-based guideline for supervisor training, which included: what training content should include, the aim (eg, behaviour modification among supervisors), frequency of training (ie, yearly) and the target population for training (eg, all supervisors). The guideline aimed to improve the mental health of workers by ensuring that all supervisors were trained and would therefore be better equipped to support the mental health of their workers.

While these reviews have made a helpful start in illustrating what effective supervision training can look like, they have their limitations. For example, these reviews do not include recently published papers (ie, papers published from 2010 onwards), they only include a small number of papers (ie, two systematic reviews with 7 and 11 controlled studies, respectively, ${ }^{33} 40$ and one narrative review which did not clearly indicate the number of papers reviewed). ${ }^{38}$ Additionally, these previous reviews tend to focus either on the mental health setting only or on supervisory support for workers' mental health, thereby failing to explore supervision training across a diverse range of health and human services professions. Furthermore, none of these reviews explore the extent to which supervision training interventions produce their effects, how, for whom and under what contexts. Therefore, the synthesis described in this protocol aims to address this gap in knowledge. Using a realist approach, this study will allow interpretability of findings across different contexts and will address the question of what supervision training interventions work, for whom, under what circumstances, how and why.

\section{Realist synthesis methodology}

Traditional methodological approaches to evaluating interventions, such as randomised controlled trials, are orientated towards conceptually simple questions of causality such as: 'is this intervention effective or not?' Such a question of causality tends to be better suited to clinical treatments rather than complex health and education interventions such as supervision training. Realist methodology, therefore, is being used increasingly to study the workings of complex health and education interventions. ${ }^{41-46}$ Realist approaches privilege context in the evaluation of interventions. For example, a realist approach acknowledges that an intervention that works well within any given healthcare service (eg, supervision training for doctors) may not work well within a human service setting in the community (eg, supervision training for housing or child protection professionals). Therefore, understanding contextual variation and how this impacts the effectiveness (or otherwise) of an intervention is crucial to the development and implementation of complex interventions. This review will be underpinned by scientific realism, which is a logic of inquiry that generates distinct research strategies and uses research methods and techniques within these to ascertain an in-depth understanding of causality. ${ }^{47}$ Such realism will help us to unpack and synthesise hidden mechanisms at play within primary studies, thus allowing us to gain deeper understandings about how supervision training as a complex programme works or fails to work and why in our chosen different settings. ${ }^{38}$

\section{Study aim and research questions}

This study aims to address the ways in which supervision training interventions impact and influence supervisory practice, for whom and under what circumstances, how and why. The research questions for this realist synthesis protocol are as follows:

1. In what ways do supervision training interventions enable or inhibit successful supervision, for whom and in what contexts?

2. What are the key mechanisms that influence supervision training outcomes?

\section{METHODS AND ANALYSIS}

The protocol for this review is registered with the International Prospective Register for Systematic Reviews (PROSPERO).

\section{Study design}

This study will be guided by Pawson's ${ }^{46}$ five stages for conducting a realist review: (1) clarifying the scope of the review; (2) determining the search strategy; (3) study selection; (4) extracting data and (5) synthesising the 
evidence and drawing conclusions. Although presented in a linear way here, the review process is iterative in nature and therefore may not follow this process precisely. The findings of this synthesis will be written up according to the Realist and MEta-narrative Evidence Synthesis: Evolving Standards (RAMESES) publication standards for realist syntheses. ${ }^{48}$

\section{Clarifying scope of the synthesis/ldentify existing theories}

The first stage of our realist synthesis will be to (a) refine the purpose of the synthesis and (b) identify any key theories to be explored. The research team, with the help of a medical librarian, will refine the purpose and identify any key theories through first scoping the current supervision training literature. This will involve creating a matrix identifying existing primary literature, literature reviews, search terms and their synonyms. The lead author will work with the medical librarian to run pilot searches through several databases to test search terms, Boolean operators and proximity searching. These pilot searches will be used to help refine the research questions based on any gaps in knowledge in the empirical literature. This scoping exercise will help in starting to identify the circumstances for supervision training use and the nature of supervision training in the health and human services workforces. While this scoping exercise may start to identify initial programme theories about how and why interventions work (or fail to work) and in what contexts, we anticipate developing programme theories through later stages of our realist synthesis (for example, see 'extraction' later).

\section{Search strategy}

A comprehensive search of the literature will be conducted in Medline (Ovid), Cumulative Index to Nursing and Allied Health Literature Plus (Ebsco), Scopus, PsycINFO (Ovid), Australian Public Affairs Information Service (Informit), Social Services Abstracts (ProQuest) and Educational Resources Information Center (ProQuest) by the lead author, with input from a medical librarian and coauthors. We do not plan to limit our searches by date. Citations and reference lists of included studies will be checked to identify additional relevant studies.

An extensive range of search terms relating to 'supervisors' or 'supervision' and 'training' (table 1) will be tested and combined with proximity searching, Boolean operators, truncations and asterisks. An example of a Medline (Ovid) search strategy is included in box 1 .

Searches will be adapted to account for the different functions of each database (see box 1 for an illustrative Medline search).

\section{Study selection}

Initial assessment of relevance will be carried out by multiple researchers. Titles and/or abstracts of studies retrieved using the search strategy, and those from additional sources against preliminary inclusion criteria, will be reviewed using the platform Covidence.

\begin{tabular}{ll} 
Table 1 Search terms to be used for the databases \\
\hline Supervisor terms & Training terms \\
\hline Supervisor*, Supervision & Education \\
Practice educator* & Professional development \\
Clinical educator* & Educating \\
Mentor, Mentoring, Mentors & Faculty development \\
Trainer $^{*}$ & 'Train the trainer' \\
Fieldwork educator $^{*}$ & Personal development \\
Instructor & CPD \\
Preceptor, preceptors & Workshop \\
Placement educator & \\
Clinical teacher* & \\
\hline
\end{tabular}

*This symbol is used to search for both plural and non-plural, for example, trainer* would search for both trainer and trainers.

The inclusion criteria for studies are as follows: (1) directly relate to one or more of the research questions; (2) relate to the following populations: health, housing, children services, youth and families services, alcohol and drug services and mental health disciplines within the health and human services workforces; (3) directly relate to supervision training interventions; (4) literature must be written in the English language; and (5) should be primary research and peer-reviewed articles only. Note that while we decided to include only peer-reviewed articles in our synthesis rather than grey literature (due to the vast literature on supervision training), we intend to employ grey literature to make sense of our synthesis findings, plus we intend to use grey literature for a subsequent realist evaluation of a supervision training programme. The key exclusion criterion is literature relating to research supervision training interventions.

The relevance of an article will be judged against the synthesis questions and in terms of whether papers can contribute to theory building. ${ }^{48} 49$ A second independent researcher will check any ambiguities at this stage (ie, articles selected as 'maybe' in Covidence). After relevance

\section{Box 1 An illustrative Medline search}

(supervisor* OR mentors OR mentor OR mentoring OR instructor* OR placement educator* OR practice educator* OR trainer* OR preceptor OR preceptors OR clinical teacher* OR clinical educator* or fieldwork educator ${ }^{\star}$ ) ADJ3 (training OR education OR educating OR workshop*) OR Supervision ADJ (training OR education OR educating OR workshop*) OR

'train the trainer*'

OR (professional development OR faculty development OR personal development OR CPD) ADJ3 (supervisor* OR mentors OR mentor OR mentoring $\mathrm{OR}$ instructor* $\mathrm{OR}$ placement educator ${ }^{\star} \mathrm{OR}$ practice educator ${ }^{\star} \mathrm{OR}$

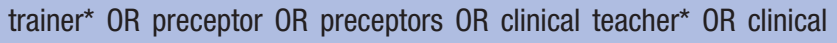
educator* ${ }^{*}$ fieldwork educator*) 


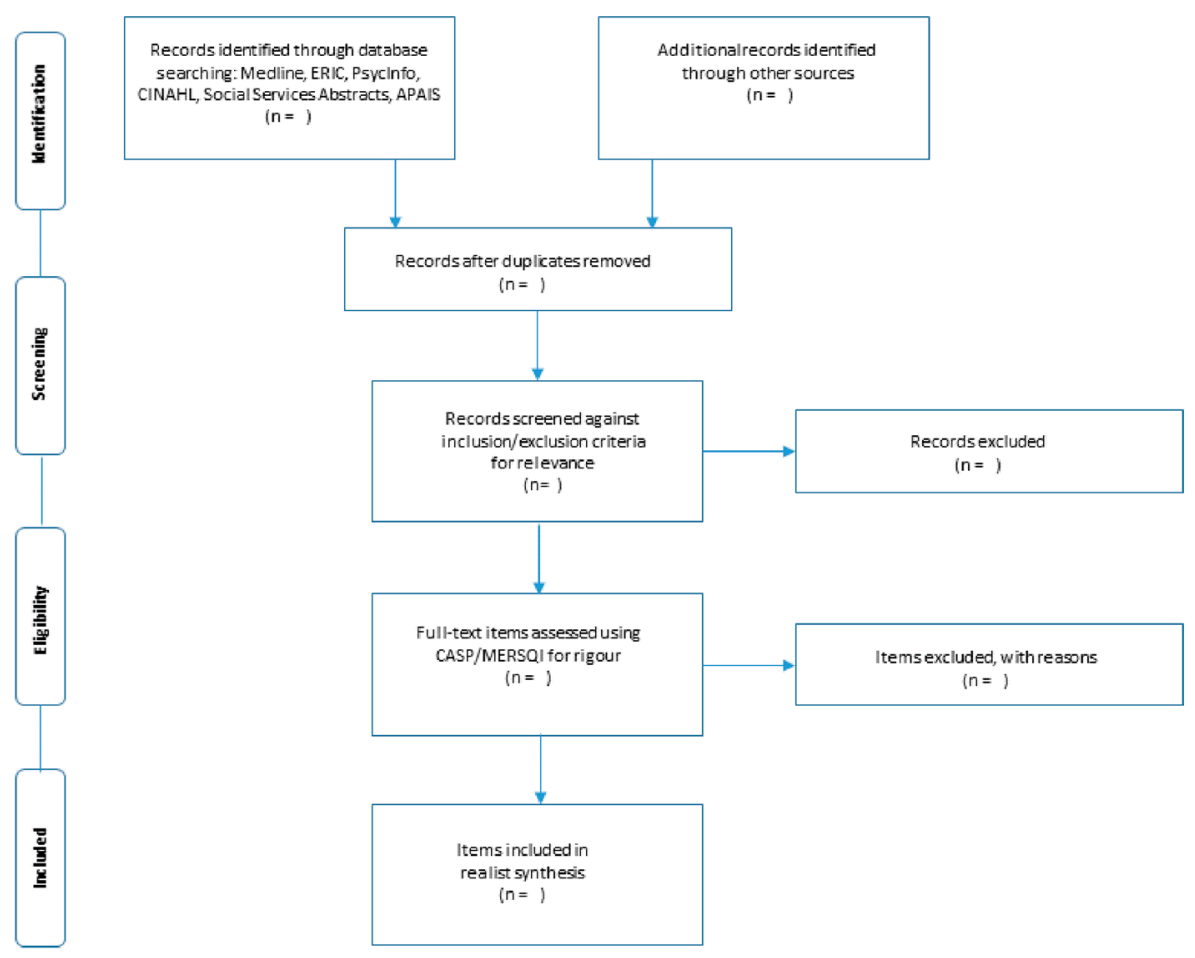

Figure 1 PRISMA diagram for realist synthesis of supervision training interventions. APAIS, Australian Public Affairs Information Service; CASP, Critical Appraisal Skills Programme; CINAHL, Cumulative Index to Nursing and Allied Health Literature; ERIC, Educational Resources Information Center; MERSQI, Medical Education Research Study Quality Instrument; PRISMA, Preferred Reporting Items for Systematic Reviews and Meta-Analyses.

checks, the full text of the remaining articles will then be retrieved and independently assessed for rigour (researchers will ask, 'are the methods used to generate data credible and trustworthy?'). ${ }^{49}$ Multiple researchers will check rigour using either the Medical Education Research Study Quality Instrument (for quantitative studies) ${ }^{50} 51$ or the Critical Appraisal Skills Programme qualitative checklist (for qualitative or mixed methods studies) ${ }^{52}$. Any disagreements over eligibility based on quality will be resolved through discussion between the researchers, and if this is not possible, an extra reviewer will be brought in to adjudicate. Double checking will be carried out and discussed for approximately $5 \%$ of included and excluded papers at the relevance stage and $10 \%$ of the included and excluded papers at the rigour stage for quality control purposes. ${ }^{53}$ The assessment of eligibility for inclusion will be an iterative process as the synthesis progresses and new or refined elements of theory may be required to explain different aspects of the developing theory/theories. Figure 1 outlines the Preferred Reporting Items for Systematic Reviews and Meta-analysis (PRISMA) process for study selection. The PRISMA diagram will be employed to allow for a clear articulation of the systematic process for this literature review.

\section{Extracting data}

An Excel spreadsheet will be used to document the extracted data from included studies. Given that this study is following a realist synthesis method, the extracted data will include: study characteristics (eg, publication year, study methodology, etc); types of participants; intervention characteristics (eg, workshop, reflective practice, seminar, etc); contexts (eg, study setting); mechanisms and outcomes and any Context-Mechanism-Outcome (CMO) configurations (CMOCs). A team of reviewers will extract data with a minimum of $10 \%$ of the papers being double checked, with any discrepancies being resolved through discussion (with a different reviewer if necessary).

The researchers will first go through the full text of each selected paper adding e-notes identifying sections of text that may be interpreted as functioning as contexts, mechanisms, outcomes and any CMOCs for each supervision training intervention. Inspired by other realist syntheses ${ }^{49}$ we aim to answer structured questions as part of this extraction process: (1) interpretation of meaning (eg, does the relevant text provide sufficient data that could be interpreted as operating as contexts, mechanisms and/or outcomes?); (2) judgements about possible CMOCs (eg, what is the partial or complete CMOC for the relevant text? Is this CMOC found elsewhere in the same or other documents?) and (3) interpretations about programme theory/theories (eg, how does this partial or full CMOC interplay with developing programme theory/theories?). This extracted data will then be transferred to the Excel spreadsheet, thus 
collating CMOs and CMOCs both within and across the sample of papers.

It is acknowledged that some articles may not fully describe or provide interventional components or contexts sufficiently, thereby not fully answering the research questions. To elicit this relevant but unpublished information, the research team will contact the study authors to request detailed information on certain aspects of interventions, particularly information on contextual factors and mechanisms that have not been reported. Such information will be added to the extraction spreadsheet if made available to authors.

\section{Synthesise findings and draw conclusions}

An Excel spreadsheet will be used to manage the data, the researchers employing realist logic analysis to interpret the data to make inferences about CMOCs and synthesise the findings. Here, we will look for any patterns across the included papers in terms of recurrent CMOCs (so-called 'demi-regularities'). ${ }^{41}$ This team-based analysis process will enable discussion of the data between researchers to allow further examination and the development of initial programme theory/theories. The process of synthesis will include: (1) comparison of findings from different studies; (2) using findings from studies to address the purpose(s) of the synthesis; (3) seeking both confirmatory and contradictory findings; (4) beginning refinement of programme theories in light of evidence and; (5) disseminating the review with findings, conclusions and recommendations. ${ }^{47}$ It is worth highlighting here that while we plan to develop initial programme theories through this realist synthesis, we will be primarily testing and modifying these initial programme theories through a subsequent realist evaluation of an Australian supervision training programme for health and human services workers.

\section{Patient and public involvement}

Given that this paper is a protocol for a realist synthesis of supervision training interventions, patients and the public were not involved in the design of the synthesis nor will they be involved in conducting and disseminating the synthesis.

\section{Potential limitations of the realist synthesis}

We acknowledge several potential limitations of the proposed realist synthesis. First, while we plan to review supervision training literature from both health and human services aligned with our funding, we recognise that these settings are wide ranging, meaning that our interpretation of context is likely to be challenging. Second, while we will pilot and refine search terms, Boolean operators and proximity searching with the assistance of a medical librarian, we will inevitably omit terms associated with supervision and/or training, for example, critical companion, coaching, facilitation and practice development, meaning that some important evidence may be missed. Third, while we have decided to include only peer-reviewed papers due to the vast supervision training literature, we realise that this excludes potentially important non peer-reviewed grey literature that could facilitate the development of initial programme theory/theories. Finally, while we have decided to employ rigour checking as part of our screening/eligibility stages like other researchers, ${ }^{42} 4345$ we know that some realist scholars do not advocate employing measures of rigour. ${ }^{54}$ While our rigour checking will help to reduce the amount of literature to be synthesised, we will prioritise the realist relevance of papers and how they can contribute to theory building.

\section{Ethics and dissemination}

Ethics approval is not required for this study as it pertains to a review of existing evidence. The findings of this synthesis will be documented using the RAMESES publication standards for realist syntheses. ${ }^{48}$ We expect to disseminate findings via a peer-reviewed journal article, conference presentation(s) and a report to the Victorian Department of Health and Human Services, Australia as the project funding body. The findings have the potential to benefit multiple stakeholders involved in developing, implementing and receiving supervision training, plus they can be used to facilitate continuous quality improvement of supervision training interventions and guide further supervision training research in the health and human services professions.

\section{Twitter Sarah Lee @MissSarahLLee and Charlotte Rees @charlreessidhu}

Acknowledgements The authors would like to thank Anne Young, Medicine, Nursing and Health Sciences Librarian from the Hargrave-Andrew Library, Monash University, for her assistance in the development of the search strategy and conduct of the searches.

Contributors All authors contributed to the protocol development. SL carried out the initial scoping exercise, while $S L$ and $C D$ wrote the first draft of the manuscript, $\mathrm{SL}, \mathrm{CP}$ and $\mathrm{CR}$ made significant revisions to the manuscript. All iterations of the manuscript were checked by VE, KP, KS, SW and BW who critically reviewed and refined the protocol further. All authors approved the final protocol. SL has been conducting this synthesis as part of a PhD, with $\mathrm{CR}$ and $\mathrm{CP}$ as her supervisors. CR is the principal investigator of the study.

Funding Monash University acknowledges the support of the Victorian Government.

Competing interests None declared.

Patient consent for publication Not required.

Provenance and peer review Not commissioned; externally peer reviewed.

Open access This is an open access article distributed in accordance with the Creative Commons Attribution Non Commercial (CC BY-NC 4.0) license, which permits others to distribute, remix, adapt, build upon this work non-commercially, and license their derivative works on different terms, provided the original work is properly cited, appropriate credit is given, any changes made indicated, and the use is non-commercial. See: http://creativecommons.org/licenses/by-nc/4.0/.

\section{REFERENCES}

1. Falender CA, Cornish JA, Goodyear R, et al. Defining competencies in psychology supervision: a consensus statement. J Clin Psychol 2004;60:771-85

2. Falender CA, Shafranske EP. Clinical supervision: a competencybased approach. Washington, DC: American Psychological Association, 2004. 
3. Kilminster SM, Jolly BC. Effective supervision in clinical practice settings: a literature review. Med Educ 2000;34:827-40.

4. Proctor B. Supervision: a co-operative exercise in accountability, enabling and ensuring. Leicester: Leicester National Youth Bureau and Council for Education and Training in Youth Work, 1986:21-4.

5. Proctor B. Supervision-competence, confidence, accountability. Brit $J$ Guid Couns 1994;22:309-18.

6. Brunero S, Stein-Parbury J. The effectiveness of clinical supervision in nursing: an evidenced based literature review. Aust $J$ Adv Nurs 2008;25:86-94.

7. Jones A. Clinical supervision: a framework for practice. Int $J$ Psychiatr Nurs Res 1996;3:290-307.

8. Knudsen HK, Ducharme LJ, Roman PM. Clinical supervision, emotional exhaustion, and turnover intention: a study of substance abuse treatment counselors in the Clinical Trials Network of the National Institute on Drug Abuse. J Subst Abuse Treat 2008;35:387-95.

9. Martin P, Copley J, Tyack Z. Twelve tips for effective clinical supervision based on a narrative literature review and expert opinion. Med Teach 2014;36:201-7.

10. Hall T, Cox D. Clinical supervision: an appropriate term for physiotherapists? Learning in Health and Social Care 2009;8:282-91.

11. Diane $\mathrm{C}$, Gonzalo A. The experience of therapy supervision within a UK multi-centre randomized controlled trial. Learning in Health and Social Care 2009;8:301-14

12. Hunter EP, Blair SEE. Staff supervision for occupational therapists. $\mathrm{Br}$ $J$ Occup Ther 1999;62:344-50.

13. Edwards D, Cooper L, Burnard P, et al. Factors influencing the effectiveness of clinical supervision. J Psychiatr Ment Health Nurs 2005;12:405-14.

14. Fowler J. Supporting self and others: from staff nurse to nurse consultant. Part 5: clinical supervision. Br J Nurs 2011;20:830-30.

15. Jones A. Clinical supervision: what do we know and what do we need to know? A review and commentary. J Nurs Manag 2006;14:577-85.

16. Dilworth S, Higgins I, Parker V, et al. Finding a way forward: a literature review on the current debates around clinical supervision. Contemp Nurse 2013;45:22-32.

17. Kleiser $\mathrm{H}, \mathrm{Cox} \mathrm{DL}$. The integration of clinical and managerial supervision: a critical literature review. Br J Occup Ther 2008;71:2-12.

18. Edwards D, Burnard P, Hannigan B, et al. Clinical supervision and burnout: the influence of clinical supervision for community mental health nurses. J Clin Nurs 2006;15:1007-15.

19. McLean M, Cilliers F, Van Wyk JM, et al. Faculty development: yesterday, today and tomorrow. Medical Teacher 2008;30:555-84.

20. Sutkin G, Wagner E, Harris I, et al. What makes a good clinical teacher in medicine? A review of the literature. Acad Med 2008;83:452-66.

21. Jochemsen-van der Leeuw HG, van Dijk N, van Etten-Jamaludin FS, et al. The attributes of the clinical trainer as a role model: a systematic review. Acad Med 2013;88:26-34.

22. Hoffman LW. The training of psychotherapy supervisors. Psychotherapy in Private Practice 1994;13:23-42.

23. Simpson S, Sparkes C. Are you getting enough? - (2) supervision models and barriers. Speech Lang Thera Prac 2008:18-19.

24. Strong J, Kavanagh D, Wilson J, et al. Supervision practice for allied health professionals within a large mental health service. Clin Superv 2004;22:191-210.

25. Geller E, Foley GM. Broadening the "ports of entry" for speechlanguage pathologists: a relational and reflective model for clinical supervision. Am J Speech Lang Pathol 2009;18:22-41.

26. Kaufman J, Schwartz T. Models of supervision: shaping professional identity. Clin Superv 2004;22:143-58.

27. Kavanagh DJ, Spence SH, Wilson J, et al. Achieving effective supervision. Drug Alcohol Rev 2002;21:247-52.

28. Siggins M. Promoting quality in clinical placements: literature review and national stakeholder consultation. Adelaide, Australia: Health Workforce Australia, 2012.

29. Davys A, Beddoe L. Best practice in professional supervision: a guide for the helping professions. London, UK: Jessica Kingsley Publishers, 2010.
30. Anderson JL. Training of supervisors in speech-language pathology and audiology. ASHA 1981;23:77-82.

31. Dowling S. Implementing the supervisory process: theory and practice. Englewood Cliffs: Prentice-Hall, 1992.

32. American Speech-Language-Hearing Association. Clinical supervision in speech-language pathology. https://www.asha.org (Accessed Jul 2008)

33. Milne DL, Sheikh Al, Pattison S, et al. Evidence-based training for clinical supervisors: a systematic review of 11 controlled studies. Clin Superv 2011;30:53-71.

34. Russell RK, Petrie T. Issues in training effective supervisors. App/ Prev Psychol 1994;3:27-42.

35. Whitman SM, Ryan B, Rubenstein DF. Psychotherapy supervisor training. Acad Psychiatry 2001;25:156-61.

36. McMahon M, Simons R. Supervision training for professional counselors: an exploratory study. Couns Educ Superv 2004;43:301-9.

37. Gaitskell S, Morley M. Supervision in occupational therapy: how are we doing? Br J Occup Ther 2008;71:119-21.

38. Gonsalvez CJ, Milne DL. Clinical supervisor training in Australia: a review of current problems and possible solutions. Aust Psychol 2010;45:233-42.

39. Bernard JM, Goodyear RK. Fundamentals of clinical supervision. 3rd ed. Needham Heights, MA, US: Allyn \& Bacon, 2004.

40. Tsutsumi A. Development of an evidence-based guideline for supervisor training in promoting mental health: literature review. $J$ Occup Health 2011;53:1-9.

41. Pawson R, Tilley N. An introduction to scientific realist evaluation. Evaluation for the 21st century: A handbook. Thousand Oaks: Sage Publications, Inc, 1997:405-18.

42. Sholl $\mathrm{S}$, Ajjawi R, Allbutt $\mathrm{H}$, et al. Balancing health care education and patient care in the UK workplace: a realist synthesis. Med Educ 2017;51:787-801.

43. Ajiawi R, Crampton PES, Rees CE. What really matters for successful research environments? A realist synthesis. Med Educ 2018:936-50.

44. Wong G, Westhorp G, Greenhalgh J, et al. Quality and reporting standards, resources, training materials and information for realist evaluation: the RAMESES II project. Health Services and Delivery Research 2017:5:1-108.

45. Kent F, Hayes J, Glass S, et al. Pre-registration interprofessional clinical education in the workplace: a realist review. Med Educ 2017;51:903-17.

46. Pawson R, Greenhalgh T, Harvey G, et al. Realist review-a new method of systematic review designed for complex policy interventions. J Health Serv Res Policy 2005;10(Suppl 1):21-34.

47. Pawson R, Greenhalgh T, Harvey G, et al; Realist synthesis: an introduction. Manchester: ESRC Research Methods Programme, University of Manchester, 2004.

48. Wong G, Greenhalgh T, Westhorp G, et al. RAMESES publication standards: realist syntheses. BMC Med 2013;11:21.

49. Abrams R, Wong G, Mahtani KR, et al. Understanding the impact of delegated home visiting services accessed via general practice by community-dwelling patients: a realist review protocol. BMJ Open 2018;8:e024876.

50. Reed DA, Beckman TJ, Wright SM. An assessment of the methodologic quality of medical education research studies published in The American Journal of Surgery. Am J Surg 2009;198:442-4.

51. Cook DA, Reed DA. Appraising the quality of medical education research methods: the Medical Education Research Study Quality Instrument and the Newcastle-Ottawa Scale-Education. Acad Med 2015;90:1067-76.

52. Critical Appraisal Skills Programme (CASP). Qualitative research checklist checklist. 2018. http://www.casp-uk.net/casp-toolschecklists/

53. Brennan N, Bryce M, Pearson M, et al. Understanding how appraisal of doctors produces its effects: a realist review protocol. BMJ Open 2014;4:e005466.

54. Wong G. Data gathering in realist reviews. Looking for needles in haystacks. In: Emmel N, Greenhalgh J, Manzano A, eds. Doing realist research. London: Sage, 2018:131-45. 